\title{
Differential Rotation of the Sun as a Source of Galactic Cosmic Rays Quasi-biennial Oscillations
}

\author{
Agnieszka Gil* \\ Siedlce University, Institute of Mathematics and Physics, Siedlce, Poland; Space Research \\ Center of Polish Academy of Sciences, Warsaw, Poland \\ E-mail: gila@uph.edu.pl

\section{Michael V. Alania} \\ Siedlce University, Faculty of Sciences, Institute of Mathematics and Physics, Siedlce, Poland; \\ Institute of Geophysics, Tbilisi State University, Tbilisi, Georgia
}

\begin{abstract}
Quasi-biennial oscillations belong to the most prominent mid-term periodicities with a duration shorter than 11-year cycle and longer than solar rotation. Sunspot number and area, intensity of coronal green line, solar radio flux, number of $\mathrm{H} \alpha$ flares, solar wind velocity, interplanetary magnetic field, as well as cosmic rays exhibit quasi-biennial oscillations in their temporal changes. However it was deeply studied, the source of the quasi-biennial oscillations is unrevealed yet. One of the concepts relates quasi-biennial oscillations to a high-frequency component of the solar dynamo functioning within the Sun's interior. Here, we propose as the origin of quasi-biennial oscillations of cosmic rays solar differential rotation.

We use 27-day averaged data of cosmic rays measured by Oulu neutron monitor and solar radio flux being a proxy of the level of solar activity. We compute wawelet coherence (WTC) in 1966-2019. Figure 1 shows that the shorter periodicities characteristic for QBO appeared clearly around 1971-1973, 1990-1993 and 1997-2003. The longest remained practically in the eighties and nineties.
\end{abstract}

36th International Cosmic Ray Conference -ICRC2019-

July 24th - August 1st, 2019

Madison, WI, U.S.A.

* Speaker. 


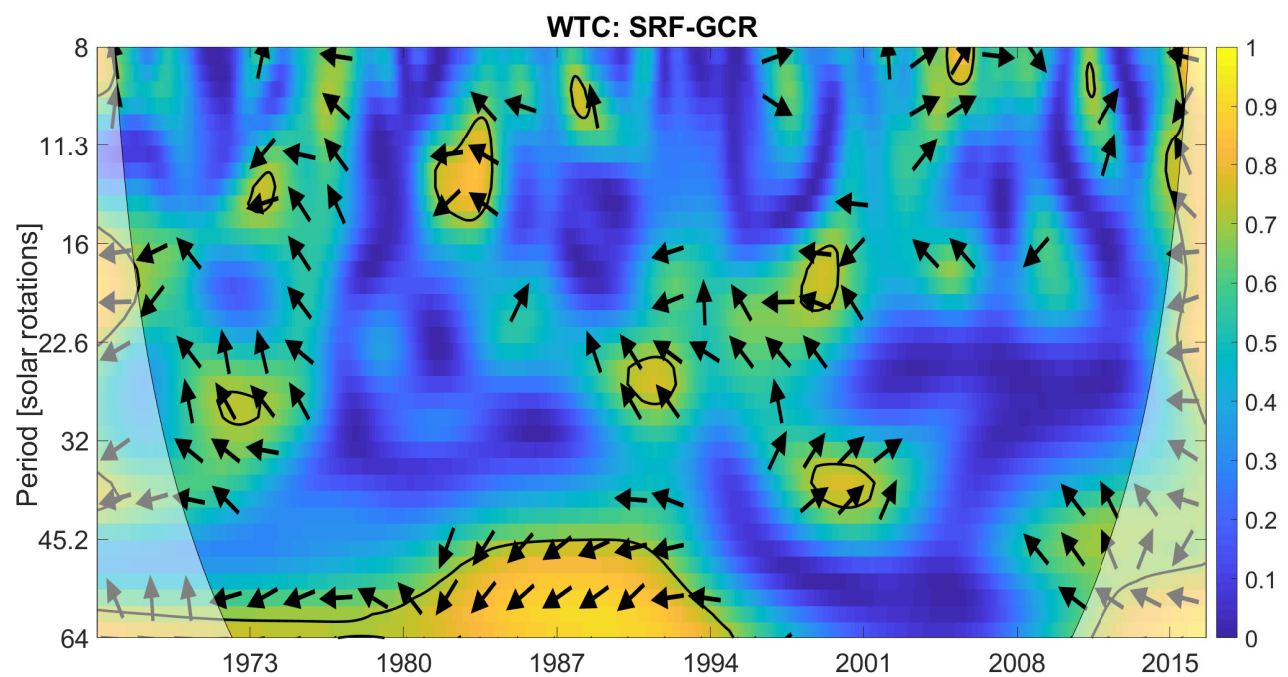

Fig.1. Wavelet coherence of cosmic rays (GCR) and solar radio flux (SRF) using the 27 days averaged data in 1966-2019. 